\title{
One-Electron Ionization of Multielectron Systems in Strong Nonresonant Laser Fields
}

\author{
Michael Spanner and Serguei Patchkovskii \\ Steacie Institute for Molecular Sciences, \\ National Research Council of Canada, Ottawa, ON, Canada K1A OR6
}

\begin{abstract}
We present a novel approach to calculating strong field ionization dynamics of multielectron molecular targets. Adopting a multielectron wavefunction ansatz based on field-free ab initio neutral and ionic multielectron states, a set of coupled time-dependent single-particle Schrödinger equations describing the neutral amplitude and continuum electron are constructed. These equations, amenable to direct numerical solution or further analytical treatment, allow one to study multielectron effects during strong field ionization, recollision, and high harmonic generation. We apply the method to strong field ionization of $\mathrm{CO}_{2}$, and suggest the importance of intermediate core excitation to explain previous failure of analytical models to reproduce experimental ionization yields for this molecule.
\end{abstract}




\section{INTRODUCTION}

Present theoretical tools for calculating strong field ionization of atoms and molecules fall into two categories, 1) semianalytical theories based the Strong Field Approximation 1] and/or ADK theory [2], often with improvements over the traditional formulation to incorporate molecular targets [3, 4], and 2) direct time-dependent numerical solution of the Schrödinger equation. The first category suffers from approximations necessary to allow a semianalytical treatment, most notably the neglect of the target-specific binding potential of the molecular core on the ionization, continuum, and recollision dynamics. The second category has the shortcoming that full numerical treatment becomes impossible as the number of degrees of freedom increases. Time-dependent numerical solutions of the Schrödinger equation including a strong laser field is only feasible for one- or two-particle systems. Steps have been made along the numerical route to incorporate multielectron effects into strong field dynamics through the use of time-dependent Hartree-Fock theory [5], multiconfigurational time-dependent Hartree-Fock [6], time-dependent configuration interaction singles [7], and time-dependent density-functional theory [8].

In this work we address both the problems of including the binding potential consistently throughout the strong field dynamics as well as the problem of accounting for a major fraction of multielectron effects. In particular, motivated by recent experiments demonstrating effects of multiple final ionic states in high harmonic generation (HHG) [9], we focus on a multiple ionic channel effects in strong field ionization which is the first step in HHG. We consider only the electronic problem, with the nuclei held fixed and work in the length gauge. Our approach to strong field ionization of multielectron targets combines ab initio quantum chemistry multielectron wavefunctions with single particle time-dependent numerical grid solutions. We use as a basis the field-free $n$-electron neutral and the lowest few $(n-1)$-electron singly ionized states. Any coupling to the multiply-charged ionic states is neglected. The wavefunction of the $n^{\text {th }}$ continuum electron associated with each ionic state is represented by a 3D Cartesian numerical grid. Equations of motion describing the evo-

lution and coupling of the basis state amplitudes and the $n^{\text {th }}$ electron wavefunction are derived from the multielectron Schrödinger equation and contain no adjustable parameters. Our method is closely related to the R-matrix theory of electron-molecular scattering [10]. We use the identical wave function ansatz. R-matrix theory accounts for antisymmetriza- 
tion exactly and is applicable to time-independent problems while our formalism includes antisymmetrization approximately but can be applied to time-dependent problems.

As a first example, we apply the method to the strong field ionization of $\mathrm{CO}_{2}$. A recent experiment [11] found that predictions made using MO-ADK for strong field ionization of $\mathrm{CO}_{2}$ failed to account for the experimental angle-resolved ionization yields. Strong field ioniozation of this molecule has also been theoretically analysed in recent papers using TDDFT in Ref.[12] and single-channel frozen-core approach in Ref.[13]. Following our analysis presented below, we suggest that an intermediate excitation channel not considered in Ref.[11] is responsible. In this channel, first an excitation of the outer-lying electron occurs concomitant with an ionic core excitation. The excited ionic core then couples back to the ground state of the inner core via laser coupling followed by release of the outer-lying electron.

\section{LENGTH GAUGE THEORY FOR ONE-ELECTRON CONTINUUM}

\section{A. Hamiltonians and States}

The (non-relativistic) Hamiltonians of the laser-free ion and neutral are

$$
\begin{gathered}
H^{I}\left(\{\vec{r}\}_{n-1}\right)=\sum_{i=1}^{n-1}\left[-\frac{1}{2} \vec{\nabla}_{i}^{2}+V_{n u c}\left(\vec{r}_{i}\right)+\sum_{j=i+1}^{n-1} \frac{1}{\left|\vec{r}_{i}-\vec{r}_{j}\right|}\right] \\
H^{N}\left(\{\vec{r}\}_{n}\right)=H^{I}\left(\{\vec{r}\}_{n-1}\right)-\frac{1}{2} \vec{\nabla}_{n}^{2}+V_{n u c}\left(\vec{r}_{n}\right)+\sum_{i=1}^{n-1} \frac{1}{\left|\vec{r}_{i}-\vec{r}_{n}\right|}
\end{gathered}
$$

where $\{\vec{r}\}_{n-1}$ are the $(n-1)$ spatial electronic coordinates of the ion, $\{\vec{r}\}_{n}$ are the $n$ spatial electronic coordinates of the neutral, and $V_{n u c}(\vec{r})$ is the electrostatic potential of the nuclei

$$
V_{\text {nuc }}(\vec{r})=\sum_{a} \frac{-Z_{a}}{\left|\vec{r}-\vec{R}_{a}\right|}
$$

where $Z_{a}$ and $\vec{R}_{a}$ are the charges and positions of the nuclei. Note that Hartree atomic units ( $\left.\hbar=m_{e}=e=1\right)$ are used throughout. In the length gauge and dipole approximation, the Hamiltonian of the full $n$ electron system interacting with the laser field is

$$
H^{F}\left(\{\vec{r}\}_{n}, t\right)=H^{N}\left(\{\vec{r}\}_{n}\right)-\sum_{i=1}^{n} \vec{F}(t) \cdot \vec{r}_{i} .
$$


Let $\left|N_{j}\right\rangle$ and $\left|I_{j}\right\rangle$ be the orthogonal $n$-electron eigenstates of the field-free neutral and the $(n-1)$-electron eigenstates of the field-free ion respectively

$$
\begin{aligned}
H^{N}\left|N_{j}\right\rangle & =E_{j}^{N}\left|N_{j}\right\rangle \\
H^{I}\left|I_{j}\right\rangle & =E_{j}^{I}\left|I_{j}\right\rangle
\end{aligned}
$$

Note that $\left|N_{j}\right\rangle$ and $\left|I_{j}\right\rangle$ depend on both spatial as well as spin coordinates of the electrons. In practice, ab initio multielectron methods provide only approximate eigenstates. The approximate nature of $\left|N_{j}\right\rangle$ and $\left|I_{j}\right\rangle$ could be taken into account by using the expectation value equations

$$
\begin{aligned}
\left\langle N_{j}\left|H^{N}\right| N_{j}\right\rangle & =E_{j}^{N} \\
\left\langle I_{j}\left|H^{I}\right| I_{j}\right\rangle & =E_{j}^{I}
\end{aligned}
$$

instead of the eigenvalue equations Eqs. (5). In this case, whenever a term like $H^{N}\left|N_{j}\right\rangle$ is encountered in the derivation, it must be replaced by the expansion

$$
H^{N}\left|N_{j}\right\rangle=\sum_{i}\left|N_{i}\right\rangle\left\langle N_{i}\left|H^{N}\right| N_{j}\right\rangle
$$

and likewise for the terms $H^{I}\left|I_{j}\right\rangle$. Thus additional terms coupling the basis states $\left|N_{j}\right\rangle$ and $\left|I_{j}\right\rangle$ will arise that are not found in the formulation when Eqs. (5) hold. For the present work it is assumed that the states are the exact neutral and ionic eigenstates and Eqs. (5) are used in the following derivation. In the following only the neutral ground state $\left|N_{0}\right\rangle=|N\rangle$ will be used.

\section{B. Antisymmetrization}

We use a wavefunction ansatz that has the form (see below for specific ansatz used)

$$
|\Psi(t)\rangle=\widehat{\mathcal{A}}\left|\Psi_{p}(t)\right\rangle,
$$

where $\left|\Psi_{p}(t)\right\rangle$ is a non-antisymmetrized 'proxy' wavefunction ansatz that treats the $n^{\text {th }}$ electron differently than the remaining $(n-1)$ core electrons,

$$
\widehat{\mathcal{A}}=\frac{1}{\sqrt{n}}\left(1-\sum_{j=1}^{n-1} \widehat{P}_{j n}\right)
$$


is the antisymmetrization operator that antisymmetrizes the $n^{\text {th }}$ electron with the remaining $(n-1)$ electrons, and $\widehat{P}_{j n}$ is the permutation operator that interchanges electrons $j$ and $n$. Note that the $(n-1)$ core electrons are already correctly antisymmetrized due to the use of fully antisymmetric $|N\rangle$ and $\left|I_{m}\right\rangle$ states. If exact propagation of $n$-electron states were possible and if the proxy wavefunction $\left|\Psi_{p}(t)\right\rangle$ spanned the full multi-electron space, the time evolution of Eq. (8) would be given by

$$
\begin{aligned}
\widehat{U}\left(t, t_{0}\right)\left|\Psi\left(t_{0}\right)\right\rangle & =\widehat{U}\left(t, t_{0}\right) \widehat{\mathcal{A}}\left|\Psi_{p}\left(t_{0}\right)\right\rangle \\
& =\widehat{U}\left(t, t_{0}\right) \frac{1}{\sqrt{n}}\left(1-\sum_{j=1}^{n-1} \widehat{P}_{j n}\right)\left|\Psi_{p}\left(t_{0}\right)\right\rangle \\
& =\frac{1}{\sqrt{n}}\left(1-\sum_{j=1}^{n-1} \widehat{P}_{j n}\right) \widehat{U}\left(t, t_{0}\right)\left|\Psi_{p}\left(t_{0}\right)\right\rangle \\
& =\widehat{\mathcal{A}} \widehat{U}\left(t, t_{0}\right)\left|\Psi_{p}\left(t_{0}\right)\right\rangle,
\end{aligned}
$$

where $\widehat{U}\left(t, t_{0}\right)$ is the evolution operator defined by

$$
i \frac{\partial}{\partial t} \widehat{U}\left(t, t_{0}\right)=H^{F}(t) \widehat{U}\left(t, t_{0}\right), \quad \widehat{U}\left(t_{0}, t_{0}\right)=\widehat{I} .
$$

Equation (10) demonstrates that, at least in the case of exact propagation, one need not propagate a fully antisymmetrized wavefunction. Rather, it is enough to propagate a partially symmetrized initial state and apply antisymmetrization at the final time: $\widehat{\mathcal{A}} \widehat{U}\left(t, t_{0}\right)\left|\Psi_{p}\left(t_{0}\right)\right\rangle$.

With this property of time evolution in mind, we proceed to construct a propagation scheme for a non-antisymmetrized proxy wavefunction ansatz

$$
\left|\Psi_{p}(t)\right\rangle=\widehat{U}\left(t, t_{0}\right)\left|\Psi_{p}\left(t_{0}\right)\right\rangle
$$

where the $n^{\text {th }}$ electron is treated differently than the $(n-1)$ core electrons. The correctly antisymmetrized wavefunction can then be retrieved using Eq. (8)). Since the propagator construct below is only approximate, due to the use of a truncated basis of ionic states, the reconstructed antisymmetric wavefunction will no longer be an exact representation of time evolution of the initial antisymmetric wavefunction. We will return to this point following the definition of $\left|\Psi_{p}(t)\right\rangle$ below to see what our propagation scheme missed using this procedure. 


\section{Projectors and Wavefunction Ansatz}

We wish to construct a propagation scheme based on coupled single-particle Schrödinger equations. With this goal in mind, we now introduce a set of single-particle orbitals that arise naturally for the present problem, and the multi-electron partitioning that will be used below.

Given the neutral ground state $|N\rangle$ and ionic states $\left|I_{m}\right\rangle$, we introduce the set of (singleparticle) orbitals, called ionization source orbitals, defined as the overlap between the neutral and ionic states

$$
\left|\phi_{m}^{S}\right\rangle=\left\langle I_{m} \mid N\right\rangle
$$

where the integration is over the $(n-1)$ electrons of the ion. These source orbitals are related to the Dyson orbitals $\left|\psi_{m}^{D}\right\rangle$ that arise in photoionization processes [14, 15] by a simple

scaling factor, $\left|\psi_{m}^{D}\right\rangle=\sqrt{n}\left|\phi_{m}^{S}\right\rangle$. In addition, it will be convenient to use the normalized source orbitals $\left|\tilde{\phi}_{m}^{S}\right\rangle$, defined as

$$
\left|\tilde{\phi}_{m}^{S}\right\rangle=\frac{\left|\phi_{m}^{S}\right\rangle}{\sqrt{\left\langle\phi_{m}^{S} \mid \phi_{m}^{S}\right\rangle}},
$$

as well as the amplitude $\eta_{m}$ :

$$
\eta_{m}=\left\langle\tilde{\phi}_{m}^{S} \mid \phi_{m}^{S}\right\rangle .
$$

Using $\left|\tilde{\phi}_{m}^{S}\right\rangle$ and its associated ionic states $\left|I_{m}\right\rangle$ we define the multi-electron source-ion states $\left|S_{m}\right\rangle$ as

$$
\left|S_{m}\right\rangle=\left|\tilde{\phi}_{m}^{S}\right\rangle\left|I_{m}\right\rangle .
$$

We now introduce the set of projectors used below to partition the multi-electron wavefunction:

$$
\begin{aligned}
\widehat{\mathcal{P}}_{m}^{S} & =\left|S_{m}\right\rangle\left\langle S_{m}\right| \\
\widehat{\mathcal{P}}^{\tilde{N}} & =|\tilde{N}\rangle\langle\tilde{N}| \\
& =\left(\widehat{I}-\sum_{k^{\prime}} \widehat{\mathcal{P}}_{k^{\prime}}^{S}\right)|N\rangle\left|\mathcal{N}_{\tilde{N}}\right|^{2}\langle N|\left(\widehat{I}-\sum_{k} \widehat{\mathcal{P}}_{k}^{S}\right) \\
\widehat{\mathcal{P}}_{m}^{I} & =\left(\widehat{I}-\widehat{\mathcal{P}}^{\tilde{N}}-\sum_{k^{\prime}} \widehat{\mathcal{P}}_{k^{\prime}}^{S}\right)\left|I_{m}\right\rangle\left\langle I_{m}\right|\left(\widehat{I}-\widehat{\mathcal{P}}^{\tilde{N}}-\sum_{k} \widehat{\mathcal{P}}_{k}^{S}\right) \\
& =\left(\widehat{I}-\widehat{\mathcal{P}}^{\tilde{N}}-\widehat{\mathcal{P}}_{m}^{S}\right)\left|I_{m}\right\rangle\left\langle I_{m}\right|\left(\widehat{I}-\widehat{\mathcal{P}}^{\tilde{N}}-\widehat{\mathcal{P}}_{m}^{S}\right)
\end{aligned}
$$


where

$$
|\tilde{N}\rangle=\mathcal{N}_{\tilde{N}}\left(\widehat{I}-\sum_{m} \widehat{\mathcal{P}}_{m}^{S}\right)|N\rangle=\mathcal{N}_{\tilde{N}}\left[|N\rangle-\sum_{m} \eta_{m}\left|S_{m}\right\rangle\right]
$$

is the (normalized) component of the neutral ground state orthogonal to the set of source-ion states $\left|S_{m}\right\rangle$ used, and

$$
\mathcal{N}_{\tilde{N}}=\left(1-\sum_{m}\left|\eta_{m}\right|^{2}\right)^{-1 / 2}
$$

is the normalization factor of the state $|\tilde{N}\rangle$. These projectors split the multi-electron space into three parts with distinct physical interpretation: the $\widehat{\mathcal{P}}_{m}^{S}$ project onto the overlap between the neutral and ionic states, $\widehat{\mathcal{P}}^{\tilde{N}}$ projects onto the component of the neutral that is orthogonal to all of the ionic states, and the $\widehat{\mathcal{P}}_{m}^{I}$ project onto the component of the ionic channels that is orthogonal to the neutral.

The projectors defined above obey the standard relations for a mutually orthogonal set of projectors

$$
\begin{aligned}
\widehat{\mathcal{P}}^{\tilde{N}} \widehat{\mathcal{P}}^{\tilde{N}} & =\widehat{\mathcal{P}}^{\tilde{N}} \\
\widehat{\mathcal{P}}_{m}^{S} \widehat{\mathcal{P}}_{k}^{S} & =\delta_{m k} \widehat{\mathcal{P}}_{m}^{S} \\
\widehat{\mathcal{P}}_{m}^{I} \widehat{\mathcal{P}}_{k}^{I} & =\delta_{m k} \widehat{\mathcal{P}}_{m}^{I} \\
\widehat{\mathcal{P}}_{m}^{S} \widehat{\mathcal{P}}^{\tilde{N}} & =\widehat{\mathcal{P}}^{\tilde{N}} \widehat{\mathcal{P}}_{m}^{S}=0 \\
\widehat{\mathcal{P}}_{m}^{S} \widehat{\mathcal{P}}_{k}^{I} & =\widehat{\mathcal{P}}_{k}^{I} \widehat{\mathcal{P}}_{m}^{S}=0 \\
\widehat{\mathcal{P}}^{\tilde{N}} \widehat{\mathcal{P}}_{m}^{I} & =\widehat{\mathcal{P}}_{m}^{I} \widehat{\mathcal{P}}^{\tilde{N}}=0
\end{aligned}
$$

where $\delta_{m k}$ is the Kronecker delta. Further, Using these relations it can be shown that

$$
\left\langle I_{m}\right| \widehat{\mathcal{P}}_{m}^{I}=\widehat{\mathcal{R}}_{m}^{S}\left\langle I_{m}\right|
$$

where $\widehat{\mathcal{R}}_{m}^{S}=\left(1-\left|\tilde{\phi}_{m}^{S}\right\rangle\left\langle\tilde{\phi}_{m}^{S}\right|\right)$ projects out (removes) the source orbital from the one-particle space connected to the $\left|I_{m}\right\rangle$ channel. Equation (21) will be used below.

The wavefunction ansatz for the proxy wavefunction constructed in the space spanned by these projectors is

$$
\left|\Psi_{p}(t)\right\rangle=b(t)|\tilde{N}\rangle+\sum_{m}\left[a_{m}(t)\left|S_{m}\right\rangle+\left|X_{m}(t)\right\rangle\right]
$$

where

$$
\left|X_{m}(t)\right\rangle=\left|\chi_{m}(t)\right\rangle\left|I_{m}\right\rangle
$$


and $\left|\chi_{m}(t)\right\rangle$ is the single-particle function that represents the excited $n^{\text {th }}$ electron associated with the ionic channel $\left|I_{m}\right\rangle$, that is, $\left|\chi_{m}(t)\right\rangle$ contains the continuum electron wavefunction that we wish to calculate. By imposing the condition $\left\langle S_{m} \mid X_{m}(t)\right\rangle=\left\langle\phi_{m}^{S} \mid \chi_{m}(t)\right\rangle=0$, which must be enforced in the initial condition and is maintained during the propagation through the use of the projection operators below, the basis states in $\left|\Psi_{p}(t)\right\rangle$ represent orthogonal spaces that can be accessed by operating with the projection operators

$$
\begin{aligned}
& \widehat{\mathcal{P}}^{\tilde{N}}\left|\Psi_{p}(t)\right\rangle=b(t)|\tilde{N}\rangle \\
& \widehat{\mathcal{P}}_{m}^{S}\left|\Psi_{p}(t)\right\rangle=a_{m}(t)\left|S_{m}\right\rangle \\
& \widehat{\mathcal{P}}_{m}^{I}\left|\Psi_{p}(t)\right\rangle=\left|\chi_{m}(t)\right\rangle\left|I_{m}\right\rangle .
\end{aligned}
$$

Returning to the issue of antisymmetrization discussion in the previous section, we can now point out the dominant interactions that are neglected using the procedure

$$
\widehat{U}\left(t, t_{0}\right) \widehat{\mathcal{A}}\left|\Psi_{p}\left(t_{0}\right)\right\rangle \rightarrow \widehat{\mathcal{A}} \widehat{U}\left(t, t_{0}\right)\left|\Psi_{p}\left(t_{0}\right)\right\rangle
$$

with the ansatz define in Eq. (22). First we note that by using fully antisymmetric neutral $|N\rangle$ and ionic states $\left|I_{m}\right\rangle$, correct antisymmetrization is present in the $(n-1)$ core electrons. Thus the procedure in Eq. (25) only concerns the $n^{\text {th }}$ (i.e. continuum) electron. When using a truncated basis of only a few low lying $\left|I_{m}\right\rangle$ states, the representation given by Eq. (22) only allows for a single electron (the $n^{\text {th }}$ electron) to be in highly excited or continuum states. Thus, no interactions that couple a continuum (or highly excited) state of one electron with a continuum state of a different electron are allowed in the present formulation. Note that these interactions are different than interactions of two electrons simultaneously in the continuum, and would appear as two-particle operators that cause transitions between twoelectron states where, for example, a continuum state of electron $j$ and a bound state of

electron $k$ simultaneously couple to a continuum states of electron $k$ and a bound state of electron $j$.

\section{Full Propagation Equations}

Consider now the Schrödinger equation for $\left|\Psi_{p}(t)\right\rangle$ (where $\partial_{t}=\partial / \partial t$ )

$$
i \partial_{t}\left|\Psi_{p}(t)\right\rangle=H^{F}(t)\left|\Psi_{p}(t)\right\rangle
$$


The solution of this equation is equivalent to solving $\widehat{U}\left(t, t_{0}\right)\left|\Psi_{p}\left(t_{0}\right)\right\rangle$ discussed above. Using the projection operators, the Schrödinger equation becomes

$$
\begin{aligned}
& i \partial_{t} \widehat{\mathcal{P}}^{\tilde{N}}\left|\Psi_{p}(t)\right\rangle=\widehat{\mathcal{P}}^{\tilde{N}} H^{F}(t) \widehat{\mathcal{P}}^{\tilde{N}}\left|\Psi_{p}(t)\right\rangle+\sum_{k} \widehat{\mathcal{P}}^{\tilde{N}} H^{F}(t) \widehat{\mathcal{P}}_{k}^{S}\left|\Psi_{p}(t)\right\rangle+\sum_{k} \widehat{\mathcal{P}}^{\tilde{N}} H^{F}(t) \widehat{\mathcal{P}}_{k}^{I} \mid \Psi_{p}((\mathbf{( 2 )}) \mathrm{a}) \\
& \left.i \partial_{t} \widehat{\mathcal{P}}_{m}^{S}\left|\Psi_{p}(t)\right\rangle=\widehat{\mathcal{P}}_{m}^{S} H^{F}(t) \widehat{\mathcal{P}}^{\tilde{N}}\left|\Psi_{p}(t)\right\rangle+\sum_{k} \widehat{\mathcal{P}}_{m}^{S} H^{F}(t) \widehat{\mathcal{P}}_{k}^{S}\left|\Psi_{p}(t)\right\rangle+\sum_{k} \widehat{\mathcal{P}}_{m}^{S} H^{F}(t) \widehat{\mathcal{P}}_{k}^{I} \mid \Psi_{p}((t)) \mathrm{b}\right) \\
& \left.i \partial_{t} \widehat{\mathcal{P}}_{m}^{I}\left|\Psi_{p}(t)\right\rangle=\widehat{\mathcal{P}}_{m}^{I} H^{F}(t) \widehat{\mathcal{P}}^{\tilde{N}}\left|\Psi_{p}(t)\right\rangle+\sum_{k} \widehat{\mathcal{P}}_{m}^{I} H^{F}(t) \widehat{\mathcal{P}}_{k}^{S}\left|\Psi_{p}(t)\right\rangle+\sum_{k} \widehat{\mathcal{P}}_{m}^{I} H^{F}(t) \widehat{\mathcal{P}}_{k}^{I} \mid \Psi_{p}((t)\rangle \mathrm{c}\right)
\end{aligned}
$$

By projecting out $|\tilde{N}\rangle,\left|S_{m}\right\rangle$, and $\left|I_{m}\right\rangle$, and recalling Eq. (21), a coupled set of Schrödinger equations for $a_{m}(t), b(t)$ and $\left|\chi_{m}(t)\right\rangle$ is obtained

$$
\begin{aligned}
i \partial_{t} b(t) & =\left\langle\tilde{N}\left|H^{F}(t)\right| \tilde{N}\right\rangle b(t)+\sum_{k}\left\langle\tilde{N}\left|H^{F}(t)\right| S_{k}\right\rangle a_{k}(t)+\sum_{k}\left\langle\tilde{N}\left|H^{F}(t)\right| X_{k}(t)\right\rangle \\
i \partial_{t} a_{m}(t) & =\left\langle S_{m}\left|H^{F}(t)\right| \tilde{N}\right\rangle b(t)+\sum_{k}\left\langle S_{m}\left|H^{F}(t)\right| S_{k}\right\rangle a_{k}(t)+\sum_{k}\left\langle S_{m}\left|H^{F}(t)\right| X_{k}(t)\right\rangle \\
i \partial_{t}\left|\chi_{m}(t)\right\rangle & =\widehat{\mathcal{R}}_{m}^{S}\left\langle I_{m}\left|H^{F}(t)\right| \tilde{N}\right\rangle b(t)+\sum_{k} \widehat{\mathcal{R}}_{m}^{S}\left\langle I_{m}\left|H^{F}(t)\right| S_{k}\right\rangle a_{k}(t)+\sum_{k} \widehat{\mathcal{R}}_{m}^{S}\left\langle I_{m}\left|H^{F}(t)\right| X_{k}(28) \phi\right)
\end{aligned}
$$

All the required matrix elements of $H^{F}(t)$ are given in the Appendix.

The set of Eqs. (28), together with the matrix elements appearing in the Appendix, is the main result of this work. In particular, they allow for the use of coupled singleparticle propagation methods to solve for the $\left|\chi_{m}(t)\right\rangle$ wavefunctions rigorously coupled to the multielectron states $|N\rangle$ and $\left|I_{m}\right\rangle$. Furthermore, numerical propagation of Eqs. (28) does not involve non-local potentials.

\section{SPECIFIC CASES AND NUMERICAL RESULTS}

\section{A. Singlet Molecules with Uncoupled Ionic Channels}

Equations (28) are completely general and can be applied to any target molecule regardless of symmetry or charge state. In this section we chose to consider the particular case of ionization from singlet molecules. Further, for simplicity in the first implementation, we consider uncoupled ionic channels. That is, we consider ionization to multiple final ionic states, but calculate ionization to each individually neglecting inter-channel couplings.

For ionization from a singlet closed-shell neutral to a particular final ion state $\left|I_{m}\right\rangle$, the ion can be left in either spin-up or spin-down states. Thus, with spin included, every final 
continuum-times-ion state has two spin-related channels, $\left|I_{m}, \uparrow\right\rangle$ and $\left|I_{m}, \downarrow\right\rangle$, each coupled to a continuum electron with opposite spin, $\left|\chi_{m}(t), \downarrow\right\rangle$ and $\left|\chi_{m}(t), \uparrow\right\rangle$ respectively. As long as any spin-orbit coupling is neglected, the two spin-related continuum functions are identical in all respects except for the differing spin label. In this case, the proxy wavefunction takes to form

$$
\begin{aligned}
|\Psi(t)\rangle= & b(t)|\tilde{N}\rangle+\left[a_{m}^{\uparrow}(t)\left|\tilde{\phi}_{m}^{S}, \uparrow\right\rangle+\left|\chi_{m}(t), \uparrow\right\rangle\right]\left|I_{m}, \downarrow\right\rangle \\
& +\left[a_{m}^{\downarrow}(t)\left|\tilde{\phi}_{m}^{S}, \downarrow\right\rangle+\left|\chi_{m}(t), \downarrow\right\rangle\right]\left|I_{m}, \uparrow\right\rangle,
\end{aligned}
$$

and Eqs. (28) reduces to

$$
\begin{aligned}
i \partial_{t} b(t) & =\mathcal{H}^{\tilde{N}}(t) b(t)+2\left\langle\mathcal{T}_{m} \mid \phi_{m}^{I}(t)\right\rangle \\
i \partial_{t} a_{m}(t) & =\left\langle\tilde { \phi } _ { m } ^ { S } \left|\left[\left|\mathcal{H}_{m}^{I}(t)\right\rangle+b(t)\left|\mathcal{T}_{m}\right\rangle\right]\right.\right. \\
i \partial_{t}\left|\chi_{m}(t)\right\rangle & =\widehat{\mathcal{R}}_{m}^{S}\left[\left|\mathcal{H}_{m}^{I}(t)\right\rangle+b(t)\left|\mathcal{T}_{m}\right\rangle\right]
\end{aligned}
$$

where $a_{m}(t)=a_{m}^{\uparrow}(t)=a_{m}^{\downarrow}(t),\left|\chi_{m}(t)\right\rangle$ represents the (identical) spatial part of $\left|\chi_{m}(t), \downarrow\right\rangle$ and $\left|\chi_{m}(t), \uparrow\right\rangle$, and

$$
\left|\phi_{m}^{I}(t)\right\rangle=\left|\chi_{m}(t)\right\rangle+a_{m}(t)\left|\tilde{\phi}_{m}^{S}\right\rangle
$$

where $\left|\tilde{\phi}_{m}^{S}\right\rangle$ is the (identical) spatial part of the two spin-related source orbitals $\left|\tilde{\phi}_{m}^{S}, \uparrow\right\rangle$ and $\left|\tilde{\phi}_{m}^{S}, \downarrow\right\rangle$. (In the following, we drop the explicit spin dependence of the states when the quantities involved to do not dependent on the spin label.) Also appearing in Eqs. (30) are

$$
\left|\mathcal{H}_{m}^{I}(t)\right\rangle=\left[H_{m}-\vec{F}(t) \cdot\left(\vec{r}_{n}-\vec{d}_{m m}^{I}\right)\right]\left|\phi_{m}^{I}(t)\right\rangle
$$

where

$$
H_{m}\left(\vec{r}_{n}\right)=E_{m}^{I}-\frac{1}{2} \vec{\nabla}_{n}^{2}+V_{n u c}\left(\vec{r}_{n}\right)+V_{m m}^{H}\left(\vec{r}_{n}\right),
$$

is the single-electron field-free Hamiltonian for the $n^{\text {th }}$ electron moving in the field of the $m^{\text {th }}$ ionic state,

$$
\vec{d}_{m m}^{I}=-\left\langle I_{m}\left|\sum_{k=1}^{n-1} \vec{r}_{k}\right| I_{m}\right\rangle
$$

is the electronic dipole moment of the ion,

$$
V_{m m}^{H}\left(\vec{r}_{n}\right)=\left\langle I_{m}\left|\sum_{k=1}^{n-1} \frac{1}{\left|\vec{r}_{k}-\vec{r}_{n}\right|}\right| I_{m}\right\rangle
$$


is the electrostatic potential of the ion core electrons. The (single particle) orbital $\left|\mathcal{T}_{m}^{\tilde{N}}\right\rangle$ defined as

$$
\left|\mathcal{T}_{m}^{\tilde{N}}\right\rangle=\mathcal{N}_{\tilde{N}}\left[\eta_{m}\left[E_{0}^{N}-\vec{F}(t) \cdot \vec{d}_{m m}^{I}-H_{m}\right]\left|\tilde{\phi}_{m}^{S}\right\rangle-\vec{F}(t) \cdot\left|\vec{\phi}_{m}^{C}\right\rangle\right]
$$

is the 'transfer orbital' that couples $\left|\tilde{\phi}_{m}^{S}\right\rangle\left|I_{m}\right\rangle$ and $\left|\chi_{m}(t)\right\rangle\left|I_{m}\right\rangle$ to the $|\tilde{N}\rangle$ component of the neutral, where $\left|\vec{\phi}_{m}^{C}\right\rangle$ is given by

$$
\left|\vec{\phi}_{m}^{C}\right\rangle=\left\langle I_{m}\left|\sum_{k=1}^{n-1} \vec{r}_{k}\right| N\right\rangle
$$

This single-particle function $\left|\vec{\phi}_{m}^{C}\right\rangle$ represents an ionization (or excitation) process where the laser field acts on a bound electron, but ionizes (or excites) a different electron. We refer to this orbital as a 'cradle orbital' in analogy with Newton's cradle, a multi-ball pendulum where one ball receives a force causing a different ball to swing. The remaining term in Eqs. (30) given by

$$
\begin{aligned}
\mathcal{H}^{\tilde{N}}(t) & =\left|\mathcal{N}_{\tilde{N}}\right|^{2}\left\{E_{0}^{N}+2\left|\eta_{m}\right|^{2}\left[\left\langle\tilde{\phi}_{m}^{S}\left|H_{m}\right| \tilde{\phi}_{m}^{S}\right\rangle-2 E_{0}^{N}\right]\right. \\
& +\vec{F}(t) \cdot\left[\vec{d}^{N}+2\left|\eta_{m}\right|^{2} \vec{d}_{m m}^{I}+2\left|\eta_{m}\right|^{2}\left\langle\tilde{\phi}_{m}^{S}\left|\vec{r}_{n}\right| \tilde{\phi}_{m}^{S}\right\rangle\right. \\
& \left.\left.+2 \eta_{m}\left\langle\vec{\phi}_{m}^{C} \mid \tilde{\phi}_{m}^{S}\right\rangle+2 \eta_{m}^{*}\left\langle\tilde{\phi}_{m}^{S} \mid \vec{\phi}_{m}^{C}\right\rangle\right]\right\}
\end{aligned}
$$

is the energy of the $|\tilde{N}\rangle$ state in the presence of the laser field, and

$$
\vec{d}^{N}=-\left\langle N\left|\sum_{k=1}^{n} \vec{r}_{k}\right| N\right\rangle
$$

is the electronic dipole moment of the neutral. The initial condition corresponding to all population in the neutral state are

$$
\begin{aligned}
b(t=0) & =\sqrt{1-2\left|\eta_{m}\right|^{2}} \\
a_{m}(t=0) & =\eta_{m} \\
\left|\chi_{m}(t=0)\right\rangle & =0
\end{aligned}
$$

The propagation equations (30) coupling the continuum electron $\left|\chi_{m}(t)\right\rangle$ to the ground state amplitudes $a_{m}(t)$ and $b(t)$ are perhaps not so transparent at first glance. They can be simplified in the case of negligible depletion and distortion of the ground state,

$$
\begin{aligned}
b(t) & \approx b(t=0) e^{-i E_{0}^{N}(t) t} \\
a_{m}(t) & \approx a_{m}(t=0) e^{-i E_{0}^{N}(t) t},
\end{aligned}
$$


where $E_{0}^{N}(t)=E_{0}^{N}-\vec{F}(t) \cdot \vec{d}^{N}-\alpha|\vec{F}(t)|^{2}$ takes into account a small Stark shift of the neutral. In this case, Eq. (30c) simplifies to

$$
\begin{aligned}
i \partial_{t}\left|\chi_{m}(t)\right\rangle & =\widehat{\mathcal{R}}_{m}^{S}\left\{\left[H_{m}-\vec{F}(t) \cdot\left(\vec{r}_{n}-\vec{d}_{m m}^{I}\right)\right]\left|\chi_{m}(t)\right\rangle\right\} \\
& +\widehat{\mathcal{R}}_{m}^{S}\left\{-\vec{F}(t) \cdot\left[\vec{r}\left|\phi_{m}^{S}\right\rangle+\left|\vec{\phi}_{m}^{C}\right\rangle\right]\right\} e^{-i E_{0}^{N}(t) t}
\end{aligned}
$$

This last equation is now very close to a standard laser-dressed single-particle Schrödinger equation for $\left|\chi_{m}(t)\right\rangle$. The only difference is that orthogonality with the neutral is maintained through the appearance of $\widehat{\mathcal{R}}_{m}^{S}$, and the term $\widehat{\mathcal{R}}_{m}^{S}\left\{-\vec{F}(t) \cdot\left[\vec{r}\left|\phi_{m}^{S}\right\rangle+\left|\vec{\phi}_{m}^{C}\right\rangle\right]\right\}$ acts as the source that populates $\left|\chi_{m}(t)\right\rangle$. For regimes where negligible depletion is expected and where Stark shifts and distortions of the neutral are small, Eqn. (42) could be used instead of Eqs. (30). In the following calculations, we use Eqs. (30) throughout.

\section{B. Ionization of $\mathrm{CO}_{2}$}

We now apply this formalism to the strong field ionization of $\mathrm{CO}_{2}$. Recently, angleresolved ionization yields have been measured [11] for this molecule, where the angle is between the molecular axis and the polarization direction of a linearly polarized laser field. In Ref. [11] it was found that the experimental angular ionization pattern for $\mathrm{CO}_{2}$ differs strongly from the results of molecular ADK theory (MO-ADK), a single-active electron quasi-static tunneling theory of molecular ionization [4]. The central difference is that MOADK predicts ionization peaks at an angle of $\sim 30^{\circ}$ while the measure show strong peaks

TABLE I: Multielectron states and energies used in the $\mathrm{CO}_{2}$ ionization calculations. Zero of energy was set equal to the (degenerate) ionic ground state.

\begin{tabular}{cccc}
\hline \hline State & Label & Energy $(\mathrm{eV})$ & Hole \\
\hline$|N\rangle$ & $\widetilde{\mathrm{X}}^{1} \Sigma_{g}$ & -13.76 & \\
$\left|I_{1}\right\rangle$ & $\widetilde{\mathrm{X}}^{2} \Pi_{g, x}$ & 0 & HOMO \\
$\left|I_{2}\right\rangle$ & $\widetilde{\mathrm{X}}^{2} \Pi_{g, y}$ & 0 & HOMO \\
$\left|I_{3}\right\rangle$ & $\widetilde{\mathrm{A}}^{2} \Pi_{u, x}$ & 3.53 & HOMO-1 \\
$\left|I_{4}\right\rangle$ & $\widetilde{\mathrm{A}}^{2} \Pi_{u, y}$ & 3.53 & HOMO-1 \\
$\left|I_{5}\right\rangle$ & $\widetilde{\mathrm{B}}^{2} \Sigma_{u}$ & 4.28 & HOMO-2 \\
\hline \hline
\end{tabular}




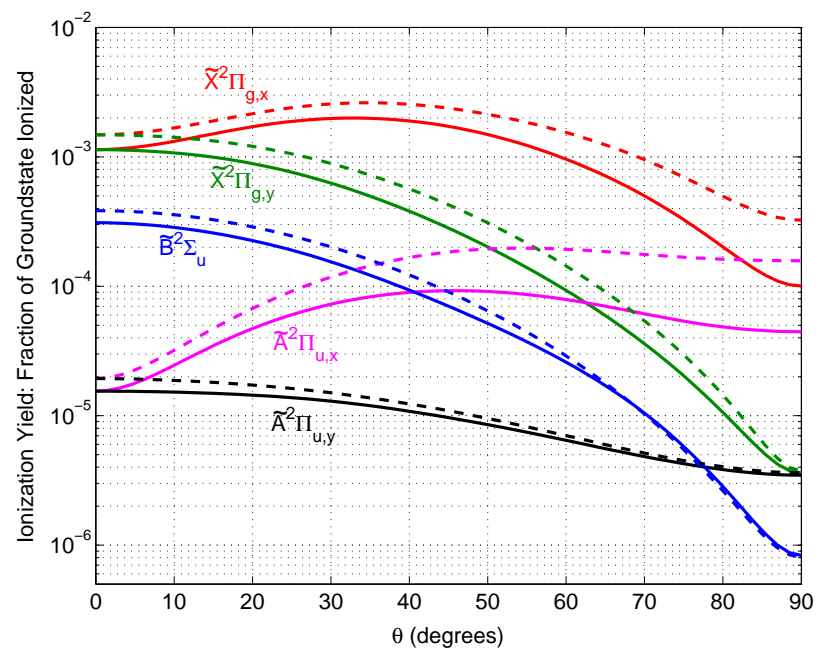

FIG. 1: (Color online) Angle-resolved ionization yields for intensity $1.5 \times 10^{14} \mathrm{~W} / \mathrm{cm}^{2}$. $\theta$ is angle between the molecular axis and the polarization axis of the laser field. The solid curves are results using a grid spacing $\Delta=0.1$ a.u. while the dashed curves used $\Delta=0.2$ a.u.

at $\sim 45^{\circ}$. Here we consider angle-resolved ionization yields of $\mathrm{CO}_{2}$ exposed to a single cycle of an $800 \mathrm{~nm}$ laser $(\omega=0.057$ a.u.).

The neutral $|N\rangle$ and lowest five ionic $\left|I_{m}\right\rangle$ multielectron orbitals are calculated using the GAMESS electronic structure code [16]. All calculations use the cc-pVTZ basis set [17] and were done at a CAS level using 16 (neutral) or 15 (cation) active electrons in 10 orbitals. Experimental geometry of the $\mathrm{CO}_{2}$ ground state is used (linear, $R_{\mathrm{C}-\mathrm{O}}=1.1621 \AA$ ). The states and energies used are shown in Table I along with the approximate location of the hole (relative to the neutral) left by the removed electron for each ionic state. Equations (29) are solved using a leapfrog algorithm. The wavefunction $\left|\chi_{m}(t)\right\rangle$ is represented on a 3-dimensional Cartesian grid. The grid extends to \pm 13 a.u. in the $\mathrm{x}$ and $\mathrm{z}$ directions, and to \pm 8 a.u. in the y direction. All calculations are done with a grid spacing of $\Delta=0.1$ a.u. in all directions unless otherwise specified. Absorbing boundary conditions are used in the xz plane with a width of 5 a.u. from the boundary edges [18]. The ionization yield was calculated by monitoring the density absorbed at the boundaries. The $\mathrm{CO}_{2}$ molecule has the $\mathrm{C}$ atom at the origin and has the bond axis aligned along the z-axis. The laser field $F(t)=\mathcal{E}_{0} \sin (\omega t)$ is rotated in the xz plane. The angle $\theta$ is the angle between the laser polarization and the molecular axis. The laser field is turned off after a single cycle, $F(t>2 \pi / \omega)=0$, and the simulations are run until $t=150$ fs (an additional 40 fs after the 
$\tilde{x}^{2} \Pi_{g, x}$

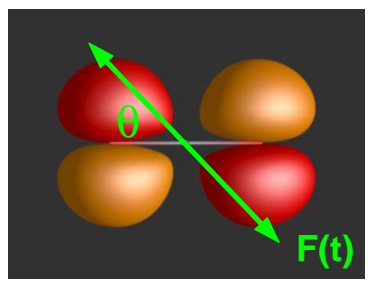

$\tilde{A}^{2} \Pi_{u, x}$

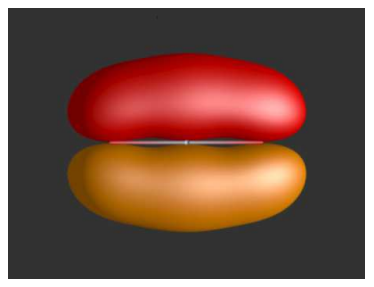

$\tilde{B}^{2} \Sigma_{u}$

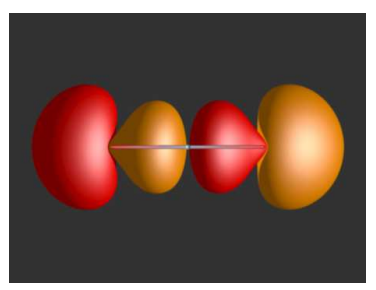

$\tilde{\mathrm{X}}^{2} \Pi_{\mathrm{g}, \mathrm{x}}$

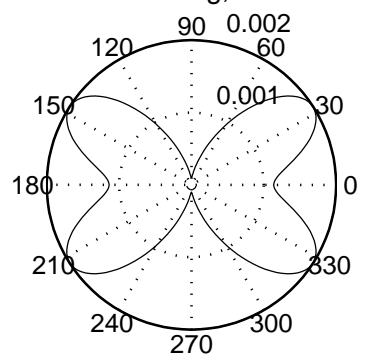

$\tilde{\mathrm{A}}^{2} \Pi_{u, x}$

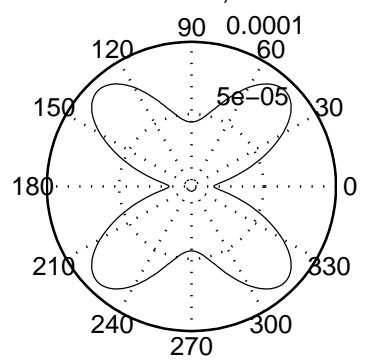

$\tilde{\mathrm{B}}^{2} \Sigma_{\mathrm{u}}$

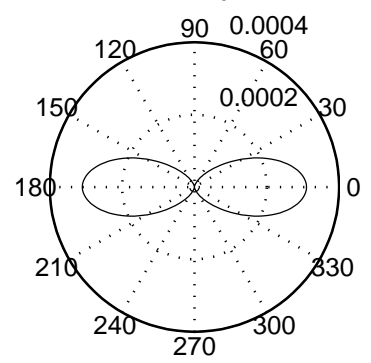

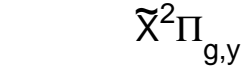

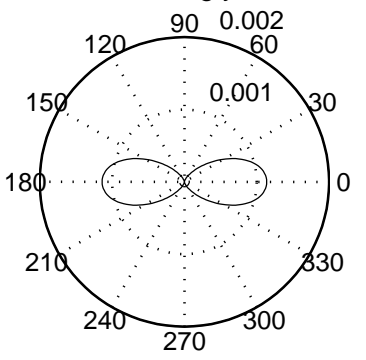

$\tilde{A}^{2} \Pi_{u, y}$

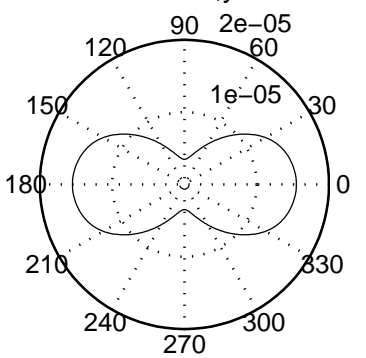

Sum of all channels

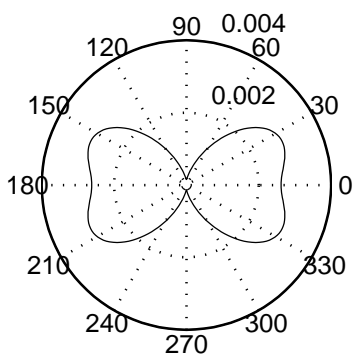

FIG. 2: (Color online) Left column: Dyson orbitals of the ionic channels considered. Angle between the laser field and the molecular axis is depicted in the top left panel. Only the Dyson orbitals with lobes in the plane of the laser field are shown. Center and right columns: Angular ionization yields for intensity $1.5 \times 10^{14} \mathrm{~W} / \mathrm{cm}^{2}$ for each ionic channel considered. Bottom-right panel shows the total ionization yield which is the sum of all channels.

single cycle is over) to allow the liberated electron density to be absorbed at the boundary. A time step of $\Delta t=0.00133$ a.u. is used for the time propagation.

Figure 1 plots angle-resolved ionization yields for the five final ion states considered for a intensity of $1.5 \times 10^{14} \mathrm{~W} / \mathrm{cm}^{2}$. The solid lines correspond to calculations with the step sizes specified above, while the dashed lines show results using $\Delta=0.2$ and $\Delta t=0.00266$ a.u. While the total yields continue to decrease a bit as the grid size becomes finer, the general character and relative behavior of the ionization channels is preserved. For all angles, the 
ionization yield is dominated by the $\widetilde{\mathrm{X}}^{2} \Pi_{g}$ channels. Polar plots showing the angular shape of each ionization channel are presented in Fig. 22. Also shown in this plot is the total ionization yield that included the yield from all channels (bottom-right panel), which is effectively the same as the yield including only the two $\widetilde{\mathrm{X}}^{2} \Pi_{g}$ channels (not shown). The total ionization yield has a 'bow tie'-like pattern, with peak values appearing near $30^{\circ}$. This is in closer agreement with the MO-ADK results than the experimental distributions, both presented in Ref. [11]. Note that the MO-ADK results of Ref. [11] include only the 'in-plane' HOMO channel which would correspond to the $\widetilde{\mathrm{X}}^{2} \Pi_{g, x}$ channel alone. Thus, our uncoupled channel calculations still fail to reproduce the experimental peak positions seen in Ref. [11].

\section{Role of Nodal Planes and the Binding Potential}

It has been shown that the presence of nodal planes in the ionizing orbitals leads to suppression of the ionization rate [3]. Most prominently, large suppression is expected to occur when the laser field is aligned along a nodal plane. This expected trend can be seen in our results (Fig. 2) by comparing the angular ionization yields with the corresponding Dyson orbitals. However, two features stand out that deserve attention. First, although suppression is seen along both nodal planes in the $\widehat{\mathrm{X}}^{2} \Pi_{g, x}$ distribution, there is much more suppression along the $90^{\circ}$ node than along the $0^{\circ}$ node. Second, there is a dip in the $\widehat{\mathrm{A}}^{2} \Pi_{u, x}$ ionization yield at $90^{\circ}$ that corresponds to no obvious feature in the $\widehat{\mathrm{A}}^{2} \Pi_{u, x}$ Dyson orbital.

Consider the $\widehat{\mathrm{X}}^{2} \Pi_{g, x}$ distribution. We first consider the case when the peak laser field is $1 \times 10^{14} \mathrm{~W} / \mathrm{cm}^{2}$ and return to the case of $1.5 \times 10^{14} \mathrm{~W} / \mathrm{cm}^{2}$ below. (Note that although the angular ionization yields shown in Fig. 2 where calculated for $1.5 \times 10^{14} \mathrm{~W} / \mathrm{cm}^{2}$, the angular shapes for each channel are very similar when using an intensity of $1 \times 10^{14} \mathrm{~W} / \mathrm{cm}^{2}$.) Panels (a) and (b) in Fig. 3 plot the $\widehat{\mathrm{X}}^{2} \Pi_{g, x}$ Dyson orbital along with select contours of the instantaneous potential at the peak of the laser pulse for an intensity of $1 \times 10^{14} \mathrm{~W} / \mathrm{cm}^{2}$. The contours are taken at the ground state energy of the neutral and show the entrance and exit of the tunneling barrier through which the $\widehat{\mathrm{X}}^{2} \Pi_{g, x}$ Dyson orbital must escape. Panel (a) shows the contours when the laser is aligned along $0^{\circ}$, while panel (b) is for the $90^{\circ}$ case. The short solid lines connecting the entrance and exit depict the tunneling path positioned along the peak of the orbitals lobes. The tunneling barrier along these paths are shown in panel (c). Already one can see that the tunneling path through which the orbital lobes must 

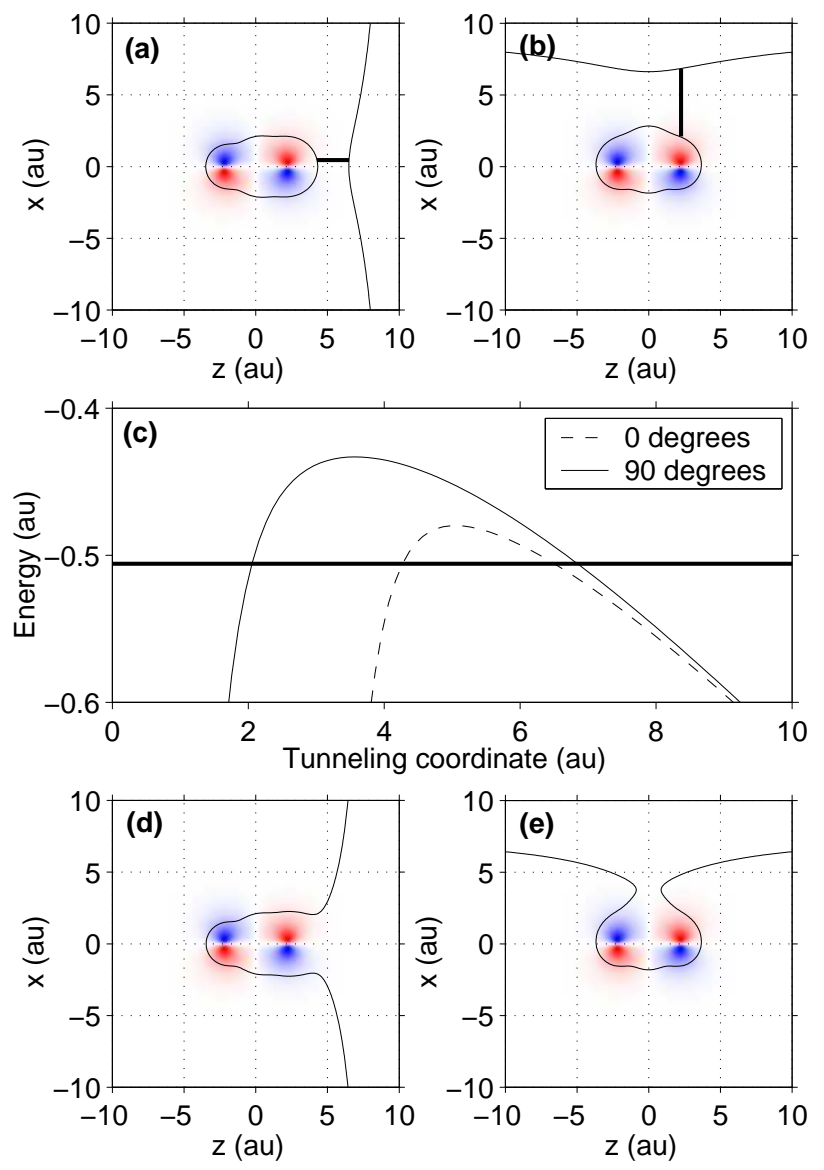

FIG. 3: (Color online) Top row: Dyson orbital of the $\widehat{\mathrm{X}}^{2} \Pi_{g, x}$ channel and ground state energy contours of the potential energy landscape at the peak of the laser field for $1 \times 10^{14} \mathrm{~W} / \mathrm{cm}^{2}$. In (a) the laser points along $0^{\circ}$ while in (b) the laser field points along $90^{\circ}$. Also shown are the tunneling paths through which the orbitals lobes must tunnel. Panel (c) plots the potential energy along the tunneling paths. The solid line denotes the ground state energy of $-13.76 \mathrm{eV}=-0.5058$ a.u. Panels (d) and (e) plot the $\widehat{\mathrm{X}}^{2} \Pi_{g, x}$ Dyson orbital along with the ground state energy contours of the potential energy for $1.5 \times 10^{14} \mathrm{~W} / \mathrm{cm}^{2}$. In panel $(\mathrm{d})$ the laser field points along $0^{o}$ while in panel (e) the laser field points along $90^{\circ}$.

pass is much shorter in the $0^{\circ}$ configuration than in the $90^{\circ}$ configuration suggesting the origin of the difference in suppression in the $0^{\circ}$ and $90^{\circ}$ degrees directions seen in the $\widehat{\mathrm{X}}^{2} \Pi_{g, x}$ distribution. In order to get a quantitative semiclassical estimate of the ratio of ionization at $0^{\circ}$ and $90^{\circ}$, we use the WKB tunneling formula

$$
\text { Rate } \sim \exp \left[-2 \int_{x_{0}}^{x_{1}} \sqrt{2\left(V\left(x^{\prime}\right)-E\right)} d x^{\prime}\right]
$$



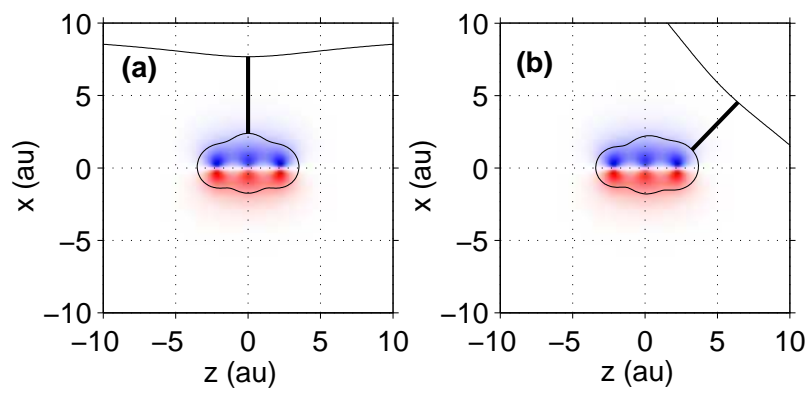

FIG. 4: (Color online) Dyson orbital of the $\widehat{\mathrm{A}}^{2} \Pi_{u, x}$ channel and ground state energy contours of the corresponding potential energy landscape at the peak of the laser field for $1.5 \times 10^{14} \mathrm{~W} / \mathrm{cm}^{2}$. In (a) the laser points along $90^{\circ}$ while in (b) the laser field points along $45^{\circ}$. The thick line segments lines show the shortest the tunneling paths.

where the integral is taken across the tunneling barrier. Using this measure, we find that the rate of tunneling along $0^{\circ}$ should be larger than the rate along $90^{\circ}$ by a factor of 7.3 , which in good agreement with the actual ratio of 8.8 extracted from the simulations. Panels (d) and (e) plot the same contours as in panels (a) and (b), but now for the intensity of $1.5 \times 10^{14} \mathrm{~W} / \mathrm{cm}^{2}$. In this case, the ionization is above barrier, and the ground state energy contours show the 'doorway' opened by the presence of the laser field. Although a quantitative estimate is difficult in the above-barrier regime, one can see that for $0^{\circ}$ the doorway encompasses almost the whole width of the Dyson orbital along this direction, while for $90^{\circ}$ the doorway is allowing only a small portion of the orbital localized around the nodal plane to pass. Thus, the analysis of the potential landscape in the $1.5 \times 10^{14} \mathrm{~W} / \mathrm{cm}^{2}$ case allows for a qualitative understanding of the large difference in suppression along the nodal planes seen in the $\widehat{\mathrm{X}}^{2} \Pi_{g, x}$ angular ionization yields.

We turn now to the $\widehat{\mathrm{A}}^{2} \Pi_{u, x}$ channel, where a similar analysis accounts for the dip at $90^{\circ}$. Figure 4 shows the $\widehat{\mathrm{A}}^{2} \Pi_{u, x}$ Dyson orbital along with the ground state energy contours. In panel (a) the laser field points along $90^{\circ}$ while panel (b) corresponds to $45^{\circ}$. Both panels correspond to a peak intensity of $1.5 \times 10^{14} \mathrm{~W} / \mathrm{cm}^{2}$, the case shown in Fig. 2. Integrating the tunneling rate along the paths shown in the plots, which are the shortest paths connecting the inner and outer regions in both cases, we calculated that the tunneling rate for the $90^{\circ}$ case should be suppressed by a factor of 0.6 as compared to the $45^{\circ}$ case. This is again in good agreement with the actual suppression of 0.5 extracted from the results in Fig. 2 for this channel. 


\section{Toward Coupled-Channel Ionization of $\mathrm{CO}_{2}$}

We can use the results of the present uncoupled channel calculations to infer potentially important ionization mechanisms that will appear in a coupled channel treatment.

In our formulation, the wavefunction $\left|\chi_{m}(t)\right\rangle$ carries not only the continuum states, but also a complete set of bound states bound to the $\left|I_{m}\right\rangle$ ionic core. Thus, using the same simulations discussed above, we can calculate excited, but un-ionized, population of $n^{\text {th }}$ electron surrounding each ionic core. In particular, the top two panels of Fig. 5 show the

angular excitation yields surrounding the $\widetilde{\mathrm{A}}^{2} \Pi_{u, x}$ ionic core for two intensities of $1 \times 10^{14}$ and $2.5 \times 10^{14} \mathrm{~W} / \mathrm{cm}^{2}$. These yields show strong peaks near (or beyond) $45^{\circ}$. In addition, as shown in Fig. 6 (a), the peak excitation yield surrounding the $\widetilde{\mathrm{A}}^{2} \Pi_{u, x}$ ionic core is much larger than the peak ionization yield coming from the $\widetilde{\mathrm{X}}^{2} \Pi_{g}$ channels.

In an uncoupled channel formulation, as is the case with the present calculations, this
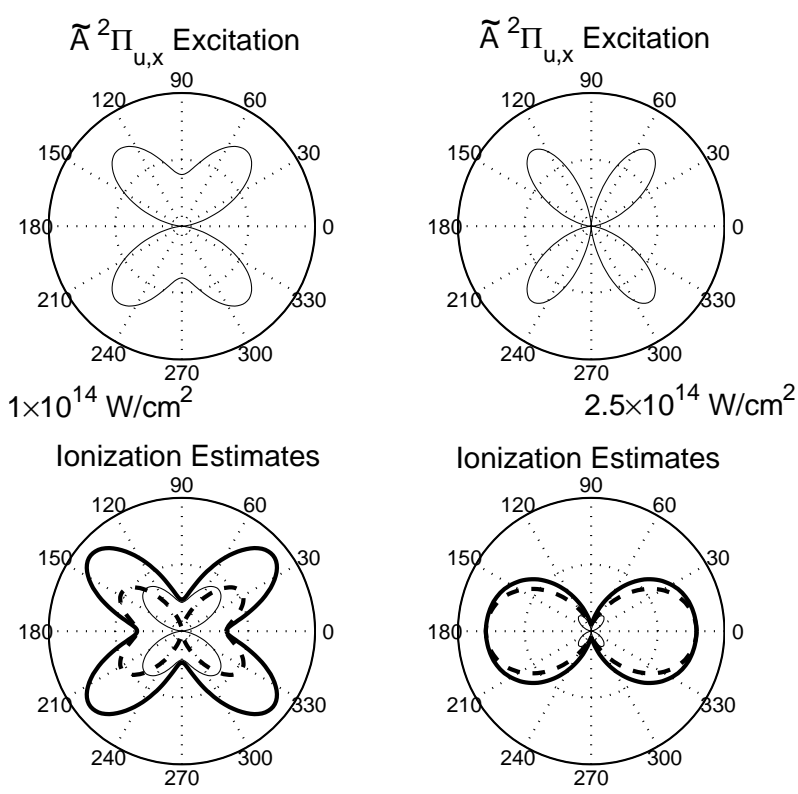

$1 \times 10^{14} \mathrm{~W} / \mathrm{cm}^{2}$

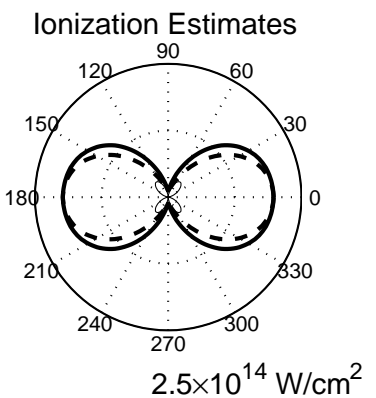

FIG. 5: Top panels: Excitation yield surrounding the $\widetilde{\mathrm{A}}^{2} \Pi_{u, x}$ ionic core for the two intensities of $1 \times 10^{14} \mathrm{~W} / \mathrm{cm}^{2}$ and $2.5 \times 10^{14} \mathrm{~W} / \mathrm{cm}^{2}$. Bottom panels: Ionization estimates for the two intensities showing the direct ionization channel (thick-dashed) analogous to the "sum over all channels" panel shown in Fig.2, the estimated intermediate excitation channel (thin), and the sum of these two channels (thick). 

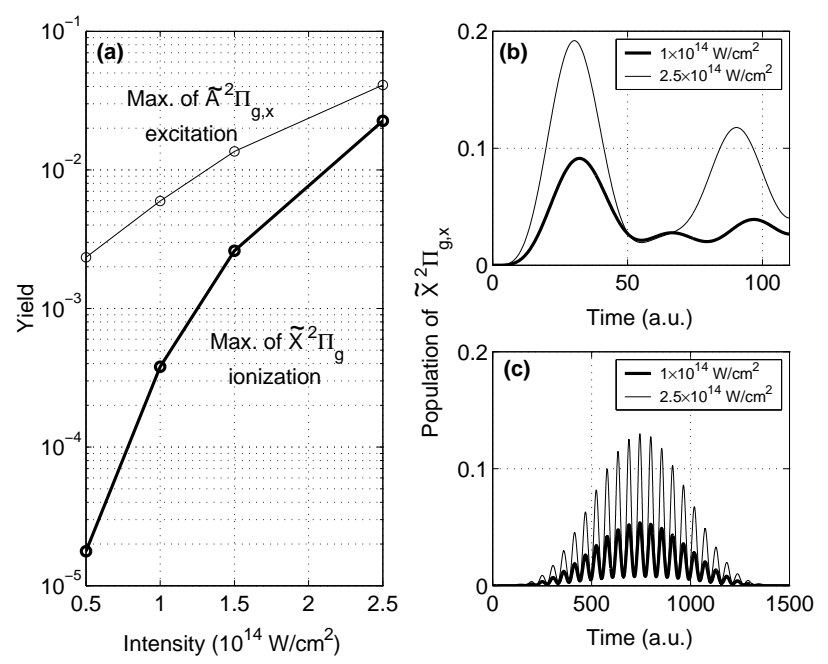

FIG. 6: Panel (a): Maximum of $\widetilde{\mathrm{A}}^{2} \Pi_{u, x}$ excitation and maximum of $\widetilde{\mathrm{X}}^{2} \Pi_{g}$ ionization as intensity is varied. Panels (b) and (c) plot the population of the $\widetilde{\mathrm{X}}^{2} \Pi_{g}$ ionic state using the 2-level laser coupled model of Eq. (44), with all population initially in the $\widetilde{\mathrm{A}}^{2} \Pi_{u, x}$ state. Panel (b) is for a single cycle pulse, and panel (c) is for a longer pulse with smooth Gaussian envelope.

excited population surrounding the $\widetilde{\mathrm{A}}^{2} \Pi_{u, x}$ ionic core is trapped. (We have checked that similar excited population exists at the end of a 5 fs Gaussian laser pulse in addition to the single cycle pulses used herein). However, in a coupled channel formulation, some of this excited population surrounding the $\widetilde{\mathrm{A}}^{2} \Pi_{u, x}$ core will be moved to the $\widetilde{\mathrm{X}}^{2} \Pi_{g, x}$ ionic core through laser-induced dipole coupling of the $\widetilde{\mathrm{A}}^{2} \Pi_{u, x}$ and $\widetilde{\mathrm{X}}^{2} \Pi_{g, x}$ core, i.e. through the polarization of the ionic cores. The amount of ionic core coupling can be estimated by solving a 2-state problem for the laser coupling of the $\widetilde{\mathrm{A}}^{2} \Pi_{u, x}$ and $\widetilde{\mathrm{X}}^{2} \Pi_{g, x}$ cores

$$
i \frac{\partial}{\partial t}\left[\begin{array}{l}
C_{X}(t) \\
C_{A}(t)
\end{array}\right]=\left[\begin{array}{cc}
E_{A} & -F(t) \mu_{A B} \\
-F(t) \mu_{A B} & E_{B}
\end{array}\right]\left[\begin{array}{l}
C_{X}(t) \\
C_{A}(t)
\end{array}\right]
$$

where $\mu_{A B}=-0.46722$ a.u. is the transition dipole between the ionic states $\widetilde{\mathrm{X}}^{2} \Pi_{g, x}$ and $\widetilde{\mathrm{A}}^{2} \Pi_{u, x}$, calculated using GAMESS as outlined above, and $C_{X}(t)$ and $C_{A}(t)$ are amplitudes of the $\widetilde{\mathrm{X}}^{2} \Pi_{g, x}$ and $\widetilde{\mathrm{A}}^{2} \Pi_{u, x}$ states. Figures 6 (b) and (c) plot $\left|C_{X}(t)\right|$ as a functions of time for two different pulse, panel (b) uses a single cycle and panel (c) uses a multi-cycle pulse with Gaussian envelope, with the initial condition $C_{X}(t)=0$ and $C_{A}(t)=1$. The calculations were done for two different intensities, $1 \times 10^{14} \mathrm{~W} / \mathrm{cm}^{2}$ (thick lines) and $2.5 \times 10^{14} \mathrm{~W} / \mathrm{cm}^{2}$ (thin lines). These calculations allows us to estimate that about 5 to $10 \%$ of the excited population surrounding the $\widetilde{\mathrm{A}}^{2} \Pi_{u, x}$ core will couple back to the $\widetilde{\mathrm{X}}^{2} \Pi_{g, x}$ state on subsequent 
cycles. Some (and perhaps all) of this excited population will escape the core region once coupled back to the $\widetilde{\mathrm{X}}^{2} \Pi_{g, x}$ ionic core. We thus anticipate two important ionization channels in a coupled-channel formulation of $\mathrm{CO}_{2}$, the direct channel and an intermediate excitation channel

$$
\begin{array}{rc}
\text { Direct : } & \mathrm{CO}_{2}\left(\widetilde{\mathrm{X}}^{1} \Sigma_{g}\right) \rightarrow \mathrm{CO}_{2}^{+}\left(\widetilde{\mathrm{X}}^{2} \Sigma_{g}\right)+e^{-} \\
\text {Inter. Ex. : } & \mathrm{CO}_{2}\left(\widetilde{\mathrm{X}}^{1} \Sigma_{g}\right) \rightarrow \mathrm{CO}_{2}^{+}\left(\widetilde{\mathrm{A}}^{2} \Sigma_{u, x}\right)\left(e^{-}\right)^{*} \rightarrow \mathrm{CO}_{2}^{+}\left(\widetilde{\mathrm{X}}^{2} \Sigma_{g}\right)+e^{-}
\end{array}
$$

where $\left(e^{-}\right)^{*}$ denotes an excited electron. Assuming that all of the excited population will escape the core upon coupling from the $\widetilde{\mathrm{A}}^{2} \Pi_{u, x}$ back to the $\widetilde{\mathrm{X}}^{2} \Pi_{g, x}$ state, the intermediate excitation channel will carry predominantly the angular imprint of the $\mathrm{CO}_{2}\left(\widetilde{\mathrm{X}}^{1} \Sigma_{g}\right) \longrightarrow$ $\mathrm{CO}_{2}^{+}\left(\widetilde{\mathrm{A}}^{2} \Sigma_{u, x}\right)\left(e^{-}\right)^{*}$ excitation step.

The direct channel yield and (estimated) intermediate channel yield, as well as their sum, is plotted in the bottom two panels of Fig. 5 for the two intensities shown. Here the intermediate excitation channel yield was estimated by multiplying the yields for excitation on the $\widetilde{\mathrm{A}}^{2} \Pi_{u, x}$ ionic core by the amount of coupling seen in Figs. 6(b) and (c), 0.05 in the case of $1 \times 10^{14} \mathrm{~W} / \mathrm{cm}^{2}$ and 0.1 for $2.5 \times 10^{14} \mathrm{~W} / \mathrm{cm}^{2}$. At the higher intensity, the direct channel dominates, while for the lower intensity the intermediate excitation channel is becoming important. Further, the peak of the total ionization estimate for $1 \times 10^{14}$ $\mathrm{W} / \mathrm{cm}^{2}$ is now approaching $45^{\circ}$, as seen in the experiment [11]. Our treatment of the proposed intermediate excitation channel is admittedly crude, and fails to reproduce the sharpness of the experimental peaks seen in Ref.[11]. An accurate description requires a full coupled-channel treatment of Eqs. (28) that includes at least the $\widetilde{\mathrm{X}}^{2} \Pi_{g, x}$ and $\widetilde{\mathrm{A}}^{2} \Pi_{u, x}$ states. However, from the scaling of the excitation and ionization yields seen in Fig. 66(a) it is clear that the intermediate excitation channel will become important for a correct description of strong field ionization of $\mathrm{CO}_{2}$ at intensities up to (and perhaps beyond) $10^{14} \mathrm{~W} / \mathrm{cm}^{2}$.

\section{SUMMARY}

In this work we developed a method for strong field one-electron ionization of multielectron targets. Our method uses field-free multielectron orbitals to describe the neutral and lowest few ionic states. These multielectron basis states are supplemented with a one-particle numerical grid used to represent the continuum electron. Equations of motion coupling the 
basis states to the continuum grid are derived from the multielectron Schrödinger equation. The result is a coupled set of single-particle Schrödinger equations describing ionization into each final ion state included in the ionic basis. Our equations are general and applicable to strong field ionization of any small molecule.

As an example, we studied ionization of $\mathrm{CO}_{2}$ in the uncoupled channel approximation including the lowest five ionic states of $\mathrm{CO}_{2}^{+}$. Strong field ionization of this molecule has been experimentally shown [11] to deviate from the predictions of MO-ADK, a single-activeelectron quasi-static model of molecular ionization. Our method allows the inclusion of two dominant effects not present in MO-ADK: 1) influence of the specific shape of the tunneling barrier discussed in Sec.III-C and 2) the possibility to rigorously couple multiple ionic channels as dissused (but presently not implementd) in Sec.III-D. In our analysis, the deviations from MO-ADK seen experimentally likely arise from intermediate ionic core excitations followed by interchannel coupling.

\section{APPENDIX A: MATRIX ELEMENTS OF THE HAMILTONIAN}

In order to evaluate the matrix elements appearing in Eq.(28), we need to know how $H^{F}\left(\left\{\vec{r}_{n}\right\}, t\right)$ acts on the basis states. The Hamiltonian acting on the neutral state gives

$$
H^{F}|N\rangle=\left(E_{0}^{N}-\sum_{k=1}^{n} \vec{F}(t) \cdot \vec{r}_{k}\right)|N\rangle .
$$

The Hamiltonian acting on a state $\left|\phi_{m}\right\rangle\left|I_{m}\right\rangle$, where $\left|I_{m}\right\rangle$ is an ionic state and $\left|\phi_{m}\right\rangle$ is here an arbitrary single particle function, gives

$$
\begin{aligned}
H^{F}\left(\left|\phi_{m}\right\rangle\left|I_{m}\right\rangle\right) & =\left[H^{I}-\frac{1}{2} \vec{\nabla}_{n}^{2}+V_{n u c}\left(\vec{r}_{n}\right)+\sum_{k=1}^{n-1} \frac{1}{\left|\vec{r}_{k}-\vec{r}_{n}\right|}-\sum_{k=1}^{n} \vec{F}(t) \cdot \vec{r}_{k}\right]\left|\phi_{m}\right\rangle\left|I_{m}\right\rangle \\
& =\sum_{j}\left|I_{j}\right\rangle\left\langle I_{j}\left|\left[E_{m}^{I}-\frac{1}{2} \vec{\nabla}_{n}^{2}+V_{n u c}\left(\vec{r}_{n}\right)+\sum_{k=1}^{n-1} \frac{1}{\left|\vec{r}_{k}-\vec{r}_{n}\right|}-\sum_{k=1}^{n} \vec{F}(t) \cdot \vec{r}_{k}\right]\right| \phi_{m}\right\rangle\left|I_{m}\right\rangle \\
& =\left(H_{m}\left(\vec{r}_{n}\right)\left|\phi_{m}\right\rangle\right)\left|I_{m}\right\rangle+\left(\sum_{j \neq m} V_{j m}^{H}\left(\vec{r}_{n}\right)\left|\phi_{m}\right\rangle\right)\left|I_{j}\right\rangle-\sum_{j}\left|I_{j}\right\rangle\left\langleI _ { j } \left|\sum_{k=1}^{n} \vec{F}(t) \cdot \vec{r}_{k}\left(\left|\phi_{m}\right\rangle\left|I_{m}\right\rangle\right)\right.\right. \\
& =\left(H_{m}\left(\vec{r}_{n}\right)\left|\phi_{m}\right\rangle\right)\left|I_{m}\right\rangle+\left(\sum_{j \neq m} V_{j m}^{H}\left(\vec{r}_{n}\right)\left|\phi_{m}\right\rangle\right)\left|I_{j}\right\rangle-\vec{F}(t) \cdot\left(\sum_{j}\left(\vec{r}_{n} \delta_{j m}-\vec{d}_{j m}^{I}\right)\left|\phi_{m}\right\rangle\right)\left|I_{j}\right\rangle
\end{aligned}
$$

where

$$
H_{m}\left(\vec{r}_{n}\right)=E_{m}^{I}-\frac{1}{2} \vec{\nabla}_{n}^{2}+V_{n u c}\left(\vec{r}_{n}\right)+V_{m m}^{H}\left(\vec{r}_{n}\right),
$$


is the single-electron field-free Hamiltonian for the $n^{\text {th }}$ electron coupled to the ionic state $\left|I_{m}\right\rangle$,

$$
\vec{d}_{j m}^{I}=-\left\langle I_{j}\left|\sum_{k=1}^{n-1} \vec{r}_{k}\right| I_{m}\right\rangle
$$

are the electronic dipole moments and transition dipoles of the ionic states, and

$$
V_{j m}^{H}\left(\vec{r}_{n}\right)=\left\langle I_{j}\left|\sum_{k=1}^{n-1} \frac{1}{\left|\vec{r}_{k}-\vec{r}_{n}\right|}\right| I_{m}\right\rangle
$$

are the electrostatic potentials and inter-ionic couplings. Eq. (A2) is only exact if a complete basis of $\left|I_{j}\right\rangle$ is used. If this basis is truncated, Eq. (因2 $)$ gives $H^{F}\left(\left|\phi_{m}\right\rangle\left|I_{m}\right\rangle\right)$ projected into the space of the truncated basis. Below we will also need the electronic dipole of the neutral defined as

$$
\vec{d}^{N}=-\left\langle N\left|\sum_{k=1}^{n} \vec{r}_{k}\right| N\right\rangle
$$

We now calculate the required matrix elements of the Hamiltonian. First First consider the matrix elements of the 'primitive' basis functions $|N\rangle,\left|S_{m}\right\rangle$, and $\left|X_{m}(t)\right\rangle$. In the following matrix elements the convention $m \neq k$ is used in order to avoid excessive use of Kronecker's delta.

$$
\begin{aligned}
\left\langle S_{m}\left|H^{F}(t)\right| S_{m}\right\rangle= & \left(\left\langle\tilde{\phi}_{m}^{S}\left|\left\langle I_{m}\right|\right) H^{F}(t)\left(\left|\tilde{\phi}_{m}^{S}\right\rangle\left|I_{m}\right\rangle\right)\right.\right. \\
= & \left\langle\tilde{\phi}_{m}^{S}\left|H_{m}\right| \tilde{\phi}_{m}^{S}\right\rangle-\vec{F}(t) \cdot\left[\left\langle\tilde{\phi}_{m}^{S}\left|\vec{r}_{n}\right| \tilde{\phi}_{m}^{S}\right\rangle-\vec{d}_{m m}^{I}\right] \\
\left\langle S_{m}\left|H^{F}(t)\right| S_{k}\right\rangle & =\left(\left\langle\tilde{\phi}_{m}^{S}\left|\left\langle I_{m}\right|\right) H^{F}(t)\left(\left|\tilde{\phi}_{k}^{S}\right\rangle\left|I_{k}\right\rangle\right)\right.\right. \\
& =\left\langle\tilde{\phi}_{m}^{S}\left|V_{m k}^{H}\right| \tilde{\phi}_{k}^{S}\right\rangle+\vec{F}(t) \cdot \vec{d}_{m k}^{I}\left\langle\tilde{\phi}_{m}^{S} \mid \tilde{\phi}_{k}^{S}\right\rangle \\
= & \eta_{m} E_{0}^{N}-\vec{F}(t) \cdot\left[\eta_{m}\left\langle\tilde{\phi}_{m}^{S}\left|\vec{r}_{n}\right| \tilde{\phi}_{m}^{S}\right\rangle+\left\langle\tilde{\phi}_{m}^{S} \mid \vec{\phi}_{m}^{C}\right\rangle\right] \\
\left\langle S_{m}\left|H^{F}(t)\right| N\right\rangle= & \left(\left\langle\tilde{\phi}_{m}^{S}\left|\left\langle I_{m}\right|\right) H^{F}(t) \mid N\right\rangle\right. \\
\left\langle S_{m}\left|H^{F}(t)\right| X_{m}(t)\right\rangle & =\left(\left\langle\tilde{\phi}_{m}^{S}\left|\left\langle I_{m}\right|\right) H^{F}(t)\left(\left|\chi_{m}(t)\right\rangle\left|I_{m}\right\rangle\right)\right.\right. \\
& =\left\langle\tilde{\phi}_{m}^{S}\left|H_{m}\right| \chi_{m}(t)\right\rangle-\vec{F}(t) \cdot\left\langle\tilde{\phi}_{m}^{S}\left|\vec{r}_{n}\right| \chi_{m}(t)\right\rangle \\
& =\left\langle\tilde{\phi}_{m}^{S}\left|V_{m k}^{H}\right| \chi_{k}(t)\right\rangle+\vec{F}(t) \cdot \vec{d}_{m k}^{I}\left\langle\tilde{\phi}_{m}^{S} \mid \chi_{k}(t)\right\rangle \\
\left\langle S_{m}\left|H^{F}(t)\right| X_{k}(t)\right\rangle= & \left(\left\langle I_{m}\right|\right) H^{F}(t)\left(\left|\chi_{k}(t)\right\rangle\left|I_{k}\right\rangle\right) \\
&
\end{aligned}
$$




$$
\begin{gathered}
\left\langle N\left|H^{F}(t)\right| N\right\rangle=E_{0}^{N}+\vec{F}(t) \cdot \vec{d}^{N} \\
\left\langle N\left|H^{F}(t)\right| X_{m}(t)\right\rangle=\left\langleN \left| H^{F}(t)\left(\left|\chi_{m}(t)\right\rangle\left|I_{m}\right\rangle\right)\right.\right. \\
=-\vec{F}(t) \cdot\left[\eta_{m}^{*}\left\langle\tilde{\phi}_{m}^{S}\left|\vec{r}_{n}\right| \chi_{m}(t)\right\rangle+\left\langle\vec{\phi}_{m}^{C} \mid \chi_{m}(t)\right\rangle\right] \\
\begin{aligned}
\left\langle I_{m}\left|H^{F}(t)\right| S_{m}\right\rangle= & \left\langleI _ { m } \left| H^{F}(t)\left(\left|\tilde{\phi}_{m}^{S}\right\rangle\left|I_{m}\right\rangle\right)\right.\right. \\
= & H_{m}\left|\tilde{\phi}_{m}^{S}\right\rangle-\vec{F}(t) \cdot\left(\vec{r}_{n}-\vec{d}_{m m}^{I}\right)\left|\tilde{\phi}_{m}^{S}\right\rangle \\
\left\langle I_{m}\left|H^{F}(t)\right| S_{k}\right\rangle=\left\langleI _ { m } \left| H^{F}(t)\left(\left|\tilde{\phi}_{k}^{S}\right\rangle\left|I_{k}\right\rangle\right)\right.\right. & =V_{m k}^{H}\left|\tilde{\phi}_{k}^{S}\right\rangle+\vec{F}(t) \cdot \vec{d}_{m k}^{I}\left|\tilde{\phi}_{k}^{S}\right\rangle \\
\left\langle I_{m}\left|H^{F}(t)\right| N\right\rangle= & \eta_{m} E_{0}^{N}\left|\tilde{\phi}_{m}^{S}\right\rangle-\vec{F}(t) \cdot\left[\vec{r}_{n}\left|\tilde{\phi}_{m}^{S}\right\rangle \eta_{m}+\left|\vec{\phi}_{m}^{C}\right\rangle\right] \\
\left\langle I_{m}\left|H^{F}(t)\right| X_{m}(t)\right\rangle= & \left\langleI _ { m } \left| H^{F}(t)\left(\left|\chi_{m}(t)\right\rangle\left|I_{m}\right\rangle\right)\right.\right. \\
= & H_{m}\left|\chi_{m}(t)\right\rangle-\vec{F}(t) \cdot\left(\vec{r}_{n}-\vec{d}_{m m}^{I}\right)\left|\chi_{m}(t)\right\rangle \\
\left\langle I_{m}\left|H^{F}(t)\right| X_{k}(t)\right\rangle= & \left\langleI _ { m } \left| H^{F}(t)\left(\left|\chi_{k}(t)\right\rangle\left|I_{k}\right\rangle\right)\right.\right. \\
& =\vec{F}_{m}(t) \cdot \vec{d}_{m k}^{I}\left|\chi_{k}(t)\right\rangle
\end{aligned} \\
=
\end{gathered}
$$

Now we use these matrix elements to evaluate the remaining terms in Eqs. (28) that involve $|\tilde{N}\rangle$

$$
\begin{aligned}
\left\langle S_{m}\left|H^{F}(t)\right| \tilde{N}\right\rangle & =\mathcal{N}_{\tilde{N}}\left[\left\langle S_{m}\left|H^{F}(t)\right| N\right\rangle-\sum_{k} \eta_{k}\left\langle S_{m}\left|H^{F}(t)\right| S_{k}\right\rangle\right] \\
& =\mathcal{N}_{\tilde{N}}\left[\left\langle S_{m}\left|H^{F}(t)\right| N\right\rangle-\eta_{m}\left\langle S_{m}\left|H^{F}(t)\right| S_{m}\right\rangle-\sum_{k}^{k \neq m} \eta_{k}\left\langle S_{m}\left|H^{F}(t)\right| S_{k}\right\rangle\right] \\
& =\mathcal{N}_{\tilde{N}}\left[\eta_{m} E_{0}^{N}-\vec{F}(t) \cdot\left[\eta_{m}\left\langle\tilde{\phi}_{m}^{S}\left|\vec{r}_{m}\right| \tilde{\phi}_{m}^{S}\right\rangle+\left\langle\tilde{\phi}_{m}^{S} \mid \vec{\phi}_{m}^{C}\right\rangle\right]\right. \\
& -\eta_{m}\left[\left\langle\tilde{\phi}_{m}^{S}\left|H_{m}\right| \tilde{\phi}_{m}^{S}\right\rangle-\vec{F}(t) \cdot\left[\left\langle\tilde{\phi}_{m}^{S}\left|\vec{r}_{m}\right| \tilde{\phi}_{m}^{S}\right\rangle-\vec{d}_{m m}^{I}\right]\right] \\
& \left.-\sum_{k \neq m}^{k \neq m} \eta_{k}\left[\left\langle\tilde{\phi}_{m}^{S}\left|V_{m k}^{H}\right| \tilde{\phi}_{k}^{S}\right\rangle+\vec{F}(t) \cdot \vec{d}_{m k}^{I}\left\langle\tilde{\phi}_{m}^{S} \mid \tilde{\phi}_{k}^{S}\right\rangle\right]\right] \\
& =\mathcal{N}_{\tilde{N}}\left[\eta_{m}\left[E_{0}^{N}-\left\langle\tilde{\phi}_{m}^{S}\left|H_{m}\right| \tilde{\phi}_{m}^{S}\right\rangle\right]-\vec{F}(t) \cdot\left[\left\langle\tilde{\phi}_{m}^{S} \mid \vec{\phi}_{m}^{C}\right\rangle+\eta_{m} \vec{d}_{m m}^{I}\right]\right. \\
& k \neq m \\
& \left.-\sum_{k}^{k \neq m} \eta_{k}\left[\left\langle\tilde{\phi}_{m}^{S}\left|V_{m k}^{H}\right| \tilde{\phi}_{k}^{S}\right\rangle+\vec{F}(t) \cdot \vec{d}_{m k}^{I}\left\langle\tilde{\phi}_{m}^{S} \mid \tilde{\phi}_{k}^{S}\right\rangle\right]\right]
\end{aligned}
$$




$$
\begin{aligned}
& \left\langle\tilde{N}\left|H^{F}(t)\right| S_{m}\right\rangle=\left\langle S_{m}\left|H^{F}(t)\right| \tilde{N}\right\rangle^{*} \\
& =\mathcal{N}_{\tilde{N}}\left[\eta_{m}^{*}\left[E_{0}^{N}-\left\langle\tilde{\phi}_{m}^{S}\left|H_{m}\right| \tilde{\phi}_{m}^{S}\right\rangle\right]-\vec{F}(t) \cdot\left[\left\langle\vec{\phi}_{m}^{C} \mid \tilde{\phi}_{m}^{S}\right\rangle+\eta_{m}^{*} \vec{d}_{m m}^{I}\right]\right. \\
& \left.-\sum_{k}^{k \neq m} \eta_{k}^{*}\left[\left\langle\tilde{\phi}_{k}^{S}\left|V_{k m}^{H}\right| \tilde{\phi}_{m}^{S}\right\rangle+\vec{F}(t) \cdot \vec{d}_{k m}^{I}\left\langle\tilde{\phi}_{k}^{S} \mid \tilde{\phi}_{m}^{S}\right\rangle\right]\right] \\
& \left\langle\tilde{N}\left|H^{F}(t)\right| \tilde{N}\right\rangle=\left|\mathcal{N}_{\tilde{N}}\right|^{2}\left(\left\langleN\left|-\sum_{m} \eta_{m}^{*}\left\langle S_{m}\right|\right) H^{F}(t)\left(|N\rangle-\sum_{k} \eta_{k}\left|S_{k}\right\rangle\right)\right.\right. \\
& =\left|\mathcal{N}_{\tilde{N}}\right|^{2}\left\langle N\left|H^{F}(t)\right| N\right\rangle+\left|\mathcal{N}_{\tilde{N}}\right|^{2} \sum_{m k}^{k \neq m} \eta_{m}^{*} \eta_{k}\left\langle S_{m}\left|H^{F}(t)\right| S_{k}\right\rangle \\
& +\left|\mathcal{N}_{\tilde{N}}\right|^{2} \sum_{m}\left[\left|\eta_{m}\right|^{2}\left\langle S_{m}\left|H^{F}(t)\right| S_{m}\right\rangle-\eta_{m}\left\langle N\left|H^{F}(t)\right| S_{m}\right\rangle-\eta_{m}^{*}\left\langle S_{m}\right| H^{F}(t)\langle\mathbb{N} \psi]\right) \\
& \left\langle\tilde{N}\left|H^{F}(t)\right| X_{m}(t)\right\rangle=\mathcal{N}_{\tilde{N}}\left[\left\langle N\left|H^{F}(t)\right| X_{m}(t)\right\rangle-\sum_{k} \eta_{k}^{*}\left\langle S_{k}\left|H^{F}(t)\right| X_{m}(t)\right\rangle\right] \\
& =\mathcal{N}_{\tilde{N}}\left[-\vec{F}(t) \cdot\left[\eta_{m}^{*}\left\langle\tilde{\phi}_{m}^{S}\left|\vec{r}_{n}\right| \chi_{m}(t)\right\rangle+\left\langle\vec{\phi}_{m}^{C} \mid \chi_{m}(t)\right\rangle\right]\right. \\
& -\eta_{m}^{*}\left[\left\langle\tilde{\phi}_{m}^{S}\left|H_{m}\right| \chi_{m}(t)\right\rangle-\vec{F}(t) \cdot\left\langle\tilde{\phi}_{m}^{S}\left|\vec{r}_{n}\right| \chi_{m}(t)\right\rangle\right] \\
& \left.-\sum_{k}^{k \neq m} \eta_{k}^{*}\left[\left\langle\tilde{\phi}_{m}^{S}\left|V_{m k}^{H}\right| \chi_{k}(t)\right\rangle+\vec{F}(t) \cdot \vec{d}_{m k}^{t}\left\langle\tilde{\phi}_{m}^{S} \mid \chi_{k}(t)\right\rangle\right]\right] \\
& =\mathcal{N}_{\tilde{N}}\left[-\eta_{m}^{*}\left\langle\tilde{\phi}_{m}^{S}\left|H_{m}\right| \chi_{m}(t)\right\rangle-\vec{F}(t) \cdot\left\langle\vec{\phi}_{m}^{C} \mid \chi_{m}(t)\right\rangle\right. \\
& \left.-\sum_{k}^{k \neq m} \eta_{k}^{*}\left[\left\langle\tilde{\phi}_{m}^{S}\left|V_{m k}^{H}\right| \chi_{k}(t)\right\rangle+\vec{F}(t) \cdot \vec{d}_{m k}^{I}\left\langle\tilde{\phi}_{m}^{S} \mid \chi_{k}(t)\right\rangle\right]\right] \\
& \left\langle I_{m}\left|H^{F}(t)\right| \tilde{N}\right\rangle=\mathcal{N}_{\tilde{N}}\left[\left\langle I_{m}\left|H^{F}(t)\right| N\right\rangle-\sum_{k} \eta_{k}\left\langle I_{m}\left|H^{F}(t)\right| S_{k}\right\rangle\right] \\
& =\mathcal{N}_{\tilde{N}}\left[E_{0}^{N}\left|\tilde{\phi}_{m}^{S}\right\rangle \eta_{m}-\vec{F}(t) \cdot \vec{r}_{n}\left|\tilde{\phi}_{m}^{S}\right\rangle \eta_{m}-\vec{F}(t) \cdot\left|\vec{\phi}_{m}^{C}\right\rangle\right. \\
& -\eta_{m} H_{m}\left|\tilde{\phi}_{m}^{S}\right\rangle+\eta_{m} \vec{F}(t) \cdot\left(\vec{r}_{n}-\vec{d}_{m m}^{I}\right)\left|\tilde{\phi}_{m}^{S}\right\rangle \\
& \left.-\sum_{k}^{k \neq m} \eta_{k}\left[V_{m k}^{H}\left|\tilde{\phi}_{k}^{S}\right\rangle+\vec{F}(t) \cdot \vec{d}_{m k}^{I}\left|\tilde{\phi}_{k}^{S}\right\rangle\right]\right] \\
& =\mathcal{N}_{\tilde{N}}\left[\eta_{m}\left[E_{0}^{N}-\vec{F}(t) \cdot \vec{d}_{m m}^{I}\right]\left|\tilde{\phi}_{m}^{S}\right\rangle-\eta_{m} H_{m}\left|\tilde{\phi}_{m}^{S}\right\rangle-\vec{F}(t) \cdot\left|\vec{\phi}_{m}^{C}\right\rangle\right. \\
& \left.-\sum_{k}^{k \neq m} \eta_{k}\left[V_{m k}^{H}\left|\tilde{\phi}_{k}^{S}\right\rangle+\vec{F}(t) \cdot \vec{d}_{m k}^{I}\left|\tilde{\phi}_{k}^{S}\right\rangle\right]\right]
\end{aligned}
$$


[1] L.V. Keldysh. Zh. Eksp. Teor. Fiz. 47, 1945 (1964) [Sov. Phys. JETP 20, 1307 (1965)]; F.H.M. Faisal. J. Phys. B 6, L89, (1973); H.R. Reiss. Phys. Rev. A 22, 1786 (1980).

[2] N.B. Delone and V.P. Krainov, Multiphoton Processes In Atoms, $2^{\text {nd }}$ ed., Springer-verlag (1994).

[3] J. Muth-Böhm, A. Becker, and F. H. M. Faisal, Phys. Rev. Lett. 85, 2280 (2000).

[4] X.M. Tong, Z.X. Zhao, and C.D. Lin, Phys. Rev. A 66 033402, (2002).

[5] K.C. Kulander, Phys. Rev. A 36, 2726 (1987); M. Lein, N. Hay, R. Velotta, J.P. Marangos, and P.L. Knight, Phys. Rev. Lett. 88, 183903 (2002).

[6] M. Kitzler, J. Zanghellini, Ch. Jungreuthmayer, M. Smits, A. Scrinzi, and T. Brabec, Phys. Rev. A 70, 041401(R) (2004); T. Kato and H. Kono, J. Chem. Phys. 128, 184102 (2008).

[7] N. Rohringer, A. Gordon, and R. Santra, Phys. Rev. A 74, 043420 (2006).

[8] D.A. Telnov and Shih-I Chu, Phys. Rev. A 79, 041401(R) (2009).

[9] W. Li, X. Zhou, R. Lock, S. Patchkovskii, A. Stolow, H.C. Kapteyn, M.M. Murnane, Science 322, 1207 (2008); B.K. McFarland, J.P. Farrell, P.H. Bucksbaum, and M. Guhr, Science 322, 1232 (2008); O. Smirnova, Y. Mairesse, S. Patchkovskii, N. Dudovich, D. Villeneuve, P. Corkum, M.Yu. Ivanov, Nature 460, 972 (2009).

[10] See, e.g., P.G. Burke and J. Tennyson, Molecular Physics 103, 2537 (2005).

[11] D. Pavičić, K.F. Lee, D.M. Rayner, P.B. Corkum, D.M. Villeneuve, Phys. Rev. Lett. 98, $243001(2007)$.

[12] Sang-Kil Son and Shih-I Chu, Phys. Rev. A 80, 011403(R) (2009).

[13] M. Abu-samha and L. B. Madsen, Phys. Rev. A 80, 023401 (2009).

[14] B.T. Pickup, Chem. Phys. 19, 192 (1977).

[15] Y. Öhrn and G. Born, Adv. Quantum Chem. 133, 1 (1981).

[16] M.W. Schmidt, K.K. Baldridge, J.A. Boatz, S.T. Elbert, M.S. Gordon, J.H. Jensen, S. Koseki, N. Matsunaga, K.A. Nguyen, S. Su, T.L. Windus, M. Dupuis, and J.A. Montgomery, J. Comput. Chem. 14, 1347 (1993).

[17] T.H. Dunning, Jr., Chem. Phys. 90, 1007 (1989).

[18] D.E. Manolopoulos, J. Chem. Phys. 117, 9552 (2002). 